\title{
Monitoring the Coldhead of Magnetic Resonance Imaging Systems by Means of Vibration Analysis
}

\author{
Jan Beyer, Johannes Krug*, Michael Friebe \\ Department for Medical Engineering, Otto-von-Guericke-University Magdeburg, Magdeburg, Germany \\ Email: *johannes.krug@ovgu.de
}

How to cite this paper: Beyer, J., Krug, J. and Friebe, M. (2017) Monitoring the Coldhead of Magnetic Resonance Imaging Systems by Means of Vibration Analysis. Journal of Sensor Technology, 7, 39-51. https://doi.org/10.4236/jst.2017.73003

Received: September 4, 2017

Accepted: September 27, 2017

Published: September 30, 2017

Copyright $\odot 2017$ by authors and Scientific Research Publishing Inc. This work is licensed under the Creative Commons Attribution International License (CC BY 4.0).

http://creativecommons.org/licenses/by/4.0/

\section{(c) (i) Open Access}

\begin{abstract}
Magnetic resonance imaging (MRI) systems require a cooling close to the absolute zero point. This is necessary to avoid thermal losses due to the extremely high currents in the coils of the electromagnet used to generate the static magnetic field. The cooling is usually achieved using helium based refrigerating machine. The coldhead is an important and critical mechanical component in this system. An inefficient or failed coldhead can lead to severe damages to the MRI system or to the loss of helium. Hence, a continuous and reliable monitoring of this system component is necessary but not always available. To tackle this problem, we propose a monitoring system by means of analyzing the structure-borne noises caused by the mechanical activities of the coldhead. For this purpose, a measurement system based on piezoelectric elements was designed and implemented. Vibrations were measured at various locations at the MRI scanner with and without MR imaging. In all positions, the function of the coldhead could be detected. Hence, the developed system is suitable for monitoring an MRI's coldhead without directly accessing the MR scanner's hardware or software. For a future long-term monitoring, the aim is to predict a failure of the MRI's coldhead based on changes in the vibrations signals.
\end{abstract}

\section{Keywords}

Machine Monitoring, Magnetic Resonance Imaging, Piezoelectric Sensors, Vibrations

\section{Introduction}

Magnetic resonance imaging (MRI) systems require a large static magnetic field. 
State of the art magnetic resonance imaging systems which are used for medical imaging purposes is operated at magnetic field strengths between $111.4 \mathrm{~T}$. The (static) magnetic field is generated by an electromagnet. Several hundred amperes are necessary to provide such high field strengths in a relatively large volume. To avoid ohmic losses within the electromagnet, the coils-which are usually made of Niob alloys-are operated in a superconducting state. To reach this state, the coils are cooled down to a temperature of $<9 \mathrm{~K}$ using a helium based cooling system [1]. Loosing the superconducting state should be avoided in all circumstance since this could result in severe damage of the MR scanner hardware and the expensive and difficult to organize helium.

To provide the cooling power, usually a helium based refrigerating machine according to the Gifford-McMahon-cycle is used [2] [3]. The machine consists of a helium compressor placed outside the MRI's RF cabin and the coldhead, which is in direct thermal contact with the parts to be cooled. The coldhead and the compressor are connected via two metallic gas pipes. The main cooling step takes place in the coldhead by an adiabatic exhaustion and cooling of the helium. Because of the extreme temperatures and the moving parts in the coldhead, this is subject to natural wear and tear. An automated monitoring of the coldhead should help to replace this only if necessary and in good time.

However, the condition of the coldhead is not directly monitored in all MRI systems. Instead, other components such as the helium level, temperatures at various locations or pressure levels are monitored by the system manufacturer. These parameters are usually not accessible for the end user or customer operating the MRI scanner system. Hence, there is an existing demand for a monitoring solution which is independent from the scanner manufacturer.

Since the coldhead is a mechanical system, it produces characteristic vibrations. These vibrations are audible inside the MRI scanner cabins. Hence, one could monitor the coldhead activity by recording audio signals inside the MRI cabin and performing an audio signal analysis. However, it is usually preferred to not perform measurements inside the MRI scanner cabin. This could be overcome by performing measurements outside the MRI cabin in the external technical installations room. Since the coldhead is mechanically connected to the outside by helium pipes, it can be assumed that the generated structure-borne noises are also transferred to the outside.

The structure-borne noises generated by mechanical systems such as rotating machines can be measured using different sensor types. The measurement of structure-borne noises is a widely practice for vibrations analysis and machine monitoring [4]. These sensors can either measure the distance, velocity or acceleration in a relative or absolute manner. The velocity is mostly measured using sensors based on electromagnetic induction principle or by Laser Doppler vibrometry [5]. Measurements of the mechanical acceleration are performed in an absolute manner using piezoelectric, piezoresistive, capacitive or optical sensor types [5]. 
In this work, we propose a monitoring system for the MRI's coldhead based on a measurement of the characteristic structure-borne noises. In general, there are two aims which are pursued: 1) detection if the coldhead is running or stopped; and 2) a long term monitoring providing information about the wear of the coldhead in order to predict a potential failure. In the present work, the focus is on the first aim since so far, no long term (several months or years) vibration data of an MRI coldhead was available. The structure-borne noises are measured at various locations outside and inside the MRI scanner cabin in order to identify suitable locations for a reliable monitoring. In addition, a robust bus system for data transmission was developed for the later application.

\section{Material and Methods}

This section describes the development of the sensor hardware and a bus system for robust sensor data transmission and acquisition. The accelerometer sensors are based on piezoelectric technology. Basic signal processing techniques were finally applied to extract and detect the mechanical vibration information for monitoring purposes.

\subsection{Piezoelectric Accelerometer}

A low-cost, disk-shaped piezoelectric element was used as sensing element (EPZ20MS64W, Ekulit GmbH, Germany). Figure 1 shows the piezoelectric accelerometer. The upper electrode and the soldering contact of the piezoelectric element form the seismic mass. This mass exerts a force proportional to the acceleration of the sensor. Due to the piezoelectric effect, this force causes a charge displacement in the piezocrystal. Using a high-impedance measuring device, a voltage proportional to the acceleration can be measured at the electrodes. The self-resonance frequency of the sensor is about $6.4 \mathrm{kHz}$.

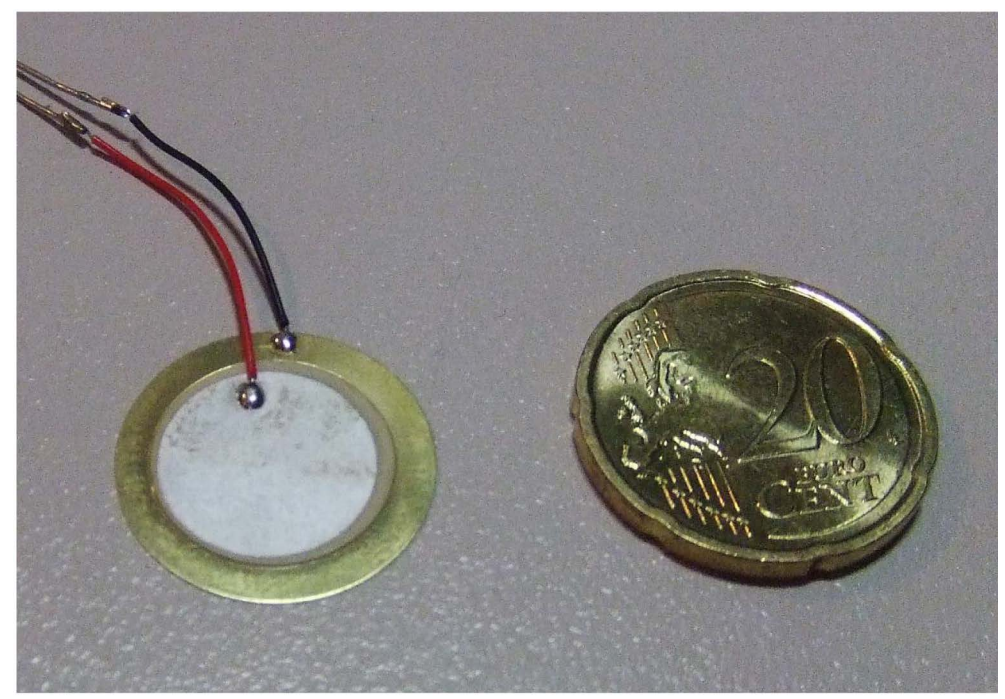

Figure 1. Piezoelectric element (left) used for the measurements of the body-born noises caused by the MRI's coldhead. 
Figure 2 shows a schematic of the analogue signal processing chain which was used to amplify and filter the signal generated by the piezoelectric element. Because the piezoelectric element is a source with a high impedance source, an amplifier with high input impedance was required. In order to obtain a high input impedance, a circuit based on MOSFET source follower with bootstrap capacitor was developed (see Figure 3). To prevent aliasing effects in the subsequent digitization of the analogue signals, a 4 order Butterworth low-pass filter with a cutoff frequency of $3 \mathrm{kHz}$ was developed (see Figure 4). For the digitization of the analogue signal, an analog-to-digital-converter (ADC) with a resolution of 12 bit was used (MC3201, Microchip Technology, USA). The ADC included a serial-peripheral-interface (SPI) which was used for the interconnection to a microcontroller. The measurements were performed at a sampling rate of 10 $\mathrm{kHz}$.

\subsection{Bus System and Data Storage}

A bus system was developed to transfer the digitized sensor data to an external measurement and data storage system. The ADC was connected to an 8-bit microcontroller (ATMega328, Microchip Technology, USA) using the SPI interface. The microcontroller was used to read the values from the ADC with a precise timing and for buffering the measured values. Therefore, an RS422-transceiver-IC (SN75179B, Texas Instruments, USA) was connected to the micro controller's

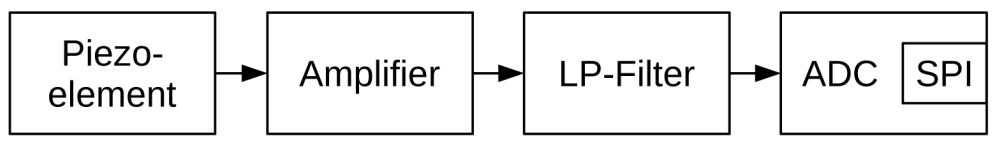

Figure 2. Schematic of the signal processing chain used to amplify, filter and digitice the analog signal of the piezoelectric element.

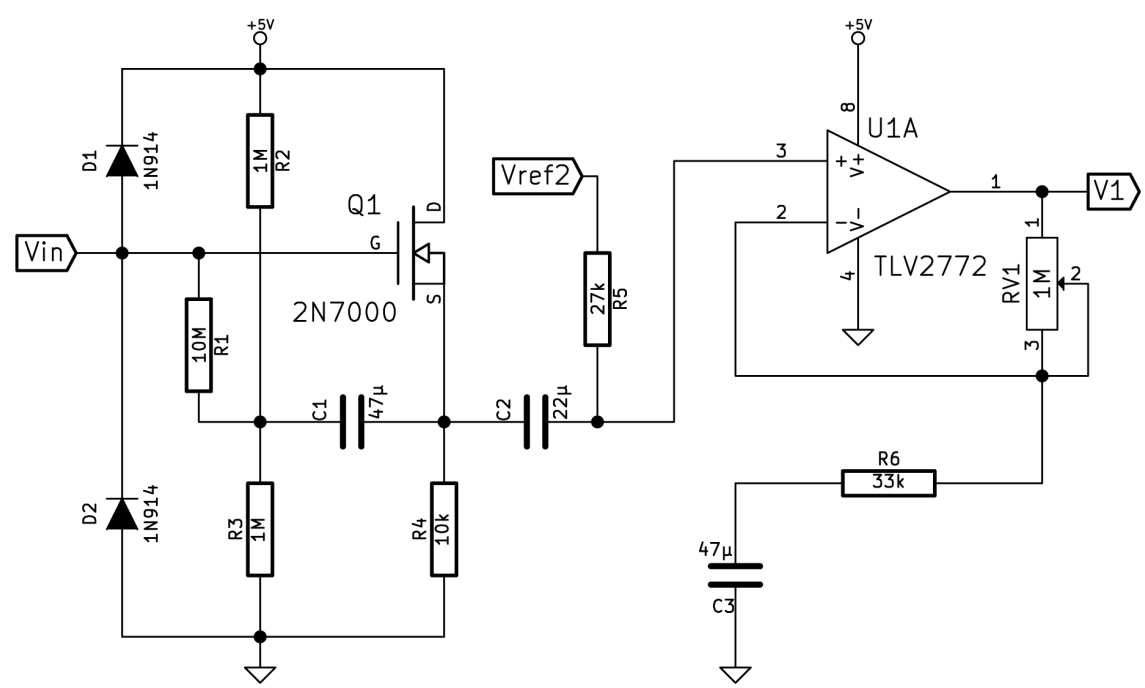

Figure 3. Preamplifier circuit the MOSFET source follower was used as a impedance converter with an amplification $\sim 1$. The actual amplifaction was achieved by the subsequent operational amplifier with an adjustable gain between 1 and 31 . 


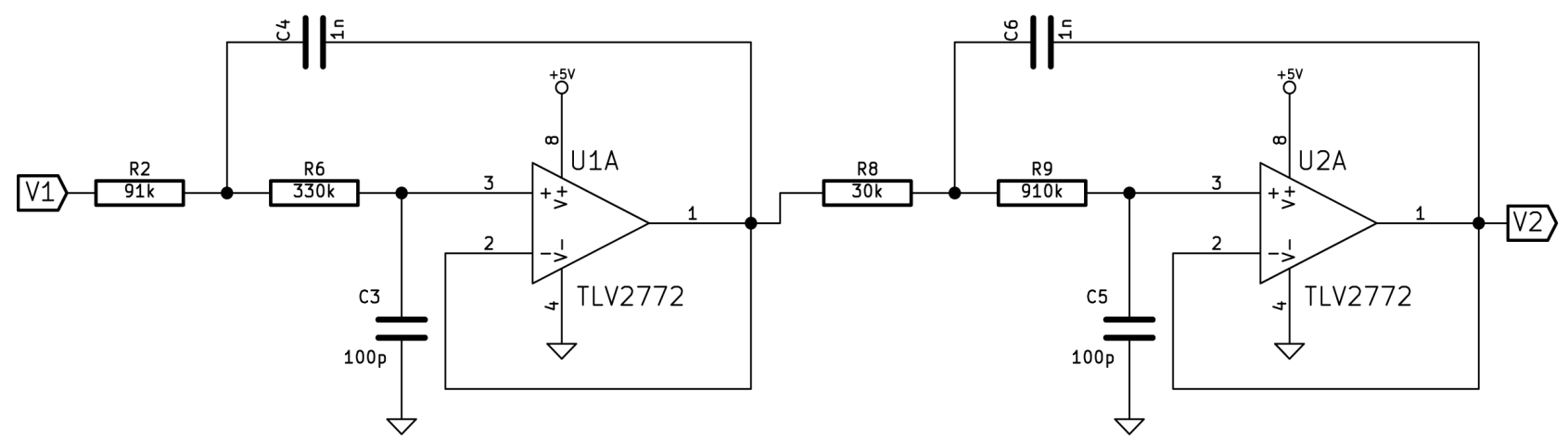

Figure 4. 4 order Butterworth filter (Sallen-Key structure) used as an anti-aliasing-filter for the subsequent digitization of the analog signal.

universal-asynchronous-receiver-transmitter (UART). The same IC was used at the master side and connected via an USB-UART converter (FT232, FTDI, Scotland) to a PC. The PC controls the communication with the sensor modules via a simple packet-oriented protocol (see Figure 5).

\subsection{Signal Processing}

The helium gas refrigerating machine according to the Gifford-McMahon-cycle works with a frequency of about $1 \mathrm{~Hz}$. The coldhead is alternately connected to the high and low pressure lines of the compressor. The flowing gas and the moving parts of the coldhead stimulate the entire structure to vibrate.

To detect whether the pump is active or not, the following signal processing was applied to the measured vibration signal. First, the signal's time-frequency power spectrum was computed using the short time Fourier transform (STFT). Depending on the frequency characteristic of the acquired signals, the power at each time step was integrated over a certain frequency range, e.g. from $23 \mathrm{kHz}$. A simple peak detection algorithm was applied to the resulting time domain signal for obtaining the operating frequency of the coldhead and for the detecting whether it is active or stopped its function.

\subsection{Measurement Setup}

The measurements were carried out on a 3T MRI system (Magnetom Skyra, Siemens, Germany). The vibrations were measured at different locations (see Figure 6). One measurement was performed directly at the surface of the coldhead (measurement point A). This position was considered as the ideal location since it is the origin of the vibrations. The other measurements were performed at the high and low pressure helium pipes in the technical equipment room which is adjacent to the MR scanner room (measurement points B-E). Points B and C were directly located behind the wall having a distance of approximately $4 \mathrm{~m}$ to the MRI's coldhead. Points D and E, which were closely located to the helium compressor, were approximately $14 \mathrm{~m}$ away from the coldhead.

Measurements of structure-borne noises were performed with MR imaging 


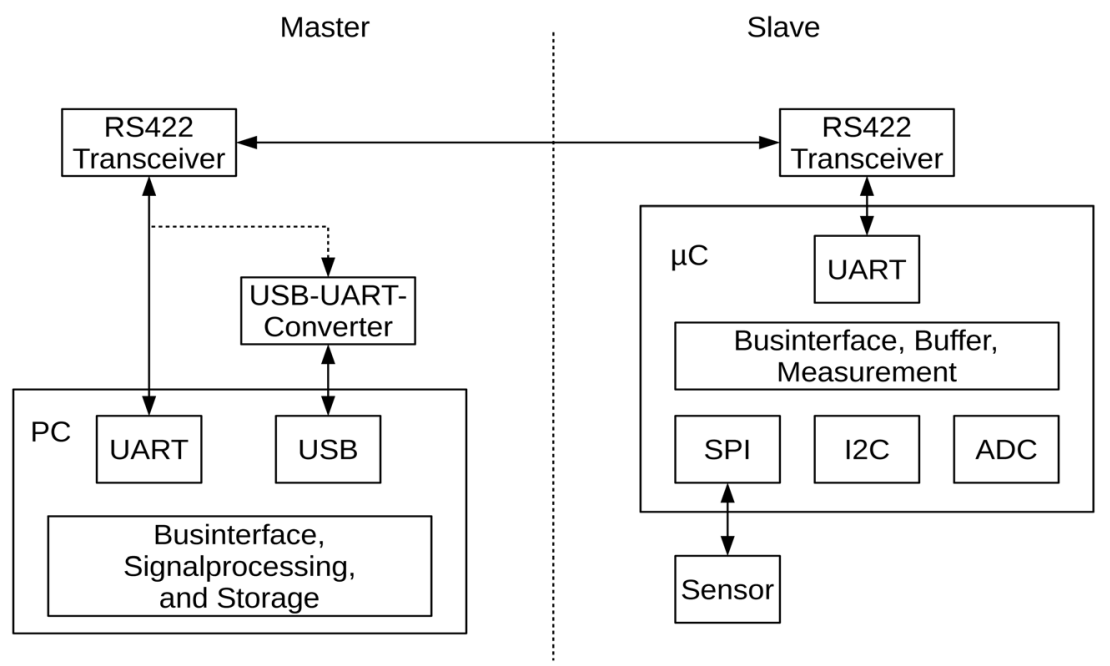

Figure 5. System architecture used for acquiring the data from the piezoelectric sensor. The master controls the function of the sensor and stores the acquired sensor values. An RS422 bus system was used to connect the master and slave modules.

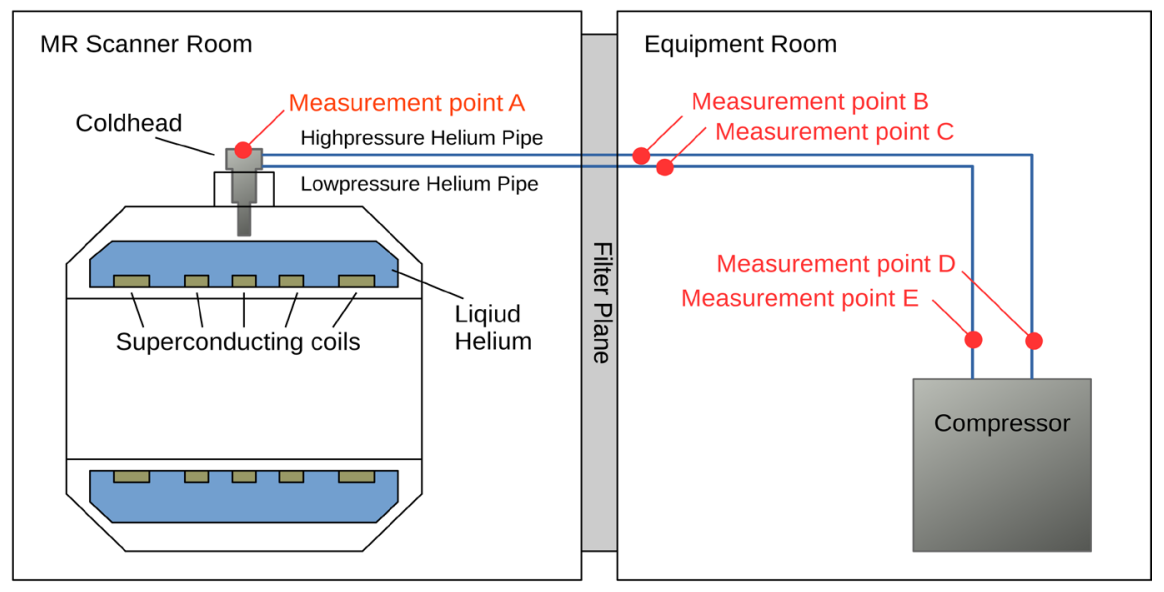

Figure 6. Principal setup of the MRI scanner and the related parts used for the vibration measurements. Measurements were performed inside the MR room directly at the coldhead (point A) or helium pipes in the adjacent technical equipment room (points B-E).

switched off and on. During MR imaging, additional mechanical vibrations are caused by the MRI's switched gradient magnetic field systems. These vibrations can potentially interfere with the vibrations of the coldhead. Hence, the influence of three different MRI sequences was studied: T2-weighted FLAIR sequence, EPI and DTI sequences [6]. It was investigated how these sequences influence the frequency spectrum of the acquired signal and if they have a negative effect on the extracted signal used for monitoring the coldhead activity.

\section{Results}

Figure 7 compares two time-domain signals which were acquired at the coldhead (measurement point $\mathrm{A}$ ) and in the equipment room (measurement point B). Both 

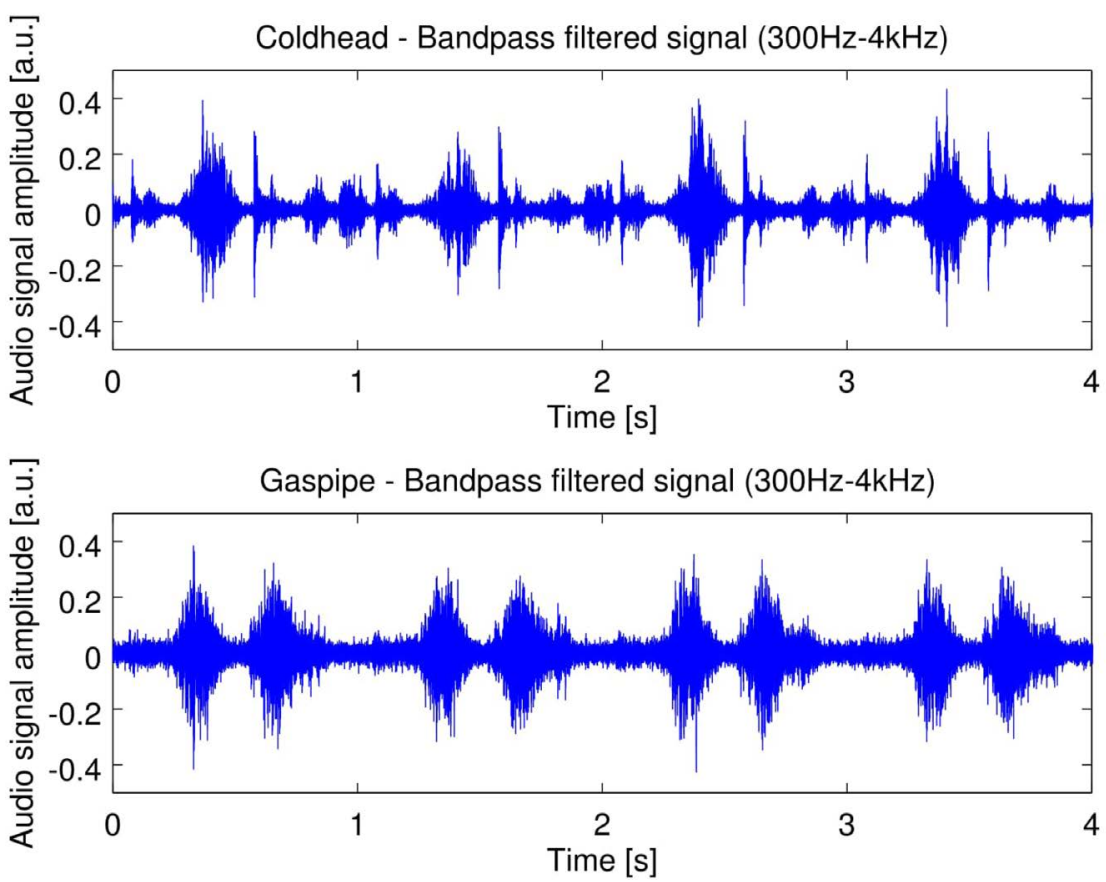

Figure 7. Vibrations measured directly at the coldhead (point A) and at the gaspipe (point B). Both signal exhibit the periodic nature of the coldhead activity whereas additional peaks with a periodicity of $2 \mathrm{~Hz}$ are visible in the coldhead signal.

band pass filtered signals show the periodic nature of the coldhead pump vibrations. The signal directly measured at the coldhead shows additional short peaks with a frequency of approximately $2 \mathrm{~Hz}$. These peaks are not directly observable in the signal measured at the helium gas pipe or were broadened by the mechanical transmission characteristics of the pipe system.

Figures 8-11 show the measured vibrations at different locations, the corresponding STFTs and the integrated power signals representing the coldhead activity. For the different locations, the main power was distributed in different frequency ranges. However, for each location, one signal could be extracted which enabled the detection of the coldhead's activity. The exemplary shown signal which was acquired during an MR imaging sequence (see Figure 11) was affected by the vibrations of the MRI's switched gradient magnetic system in the frequency range between $1 \mathrm{kHz}$ and $2 \mathrm{kHz}$. However, these distortions had no negative influence on the extracted coldhead activity signal.

\section{Discussion}

This work proposed a measurement principle and corresponding experimental setup for monitoring an MRI's coldhead activity. The vibrations and the resulting structure-borne noise caused by the coldhead were measured at various locations. From the processed vibration measurements, a signal was extracted which represented the coldhead activity and which was used to detect whether the coldhead is active or note (e.g. due to a failure) and at which frequency the coldhead is operating (usually approximately $1 \mathrm{~Hz}$. All the locations which were 


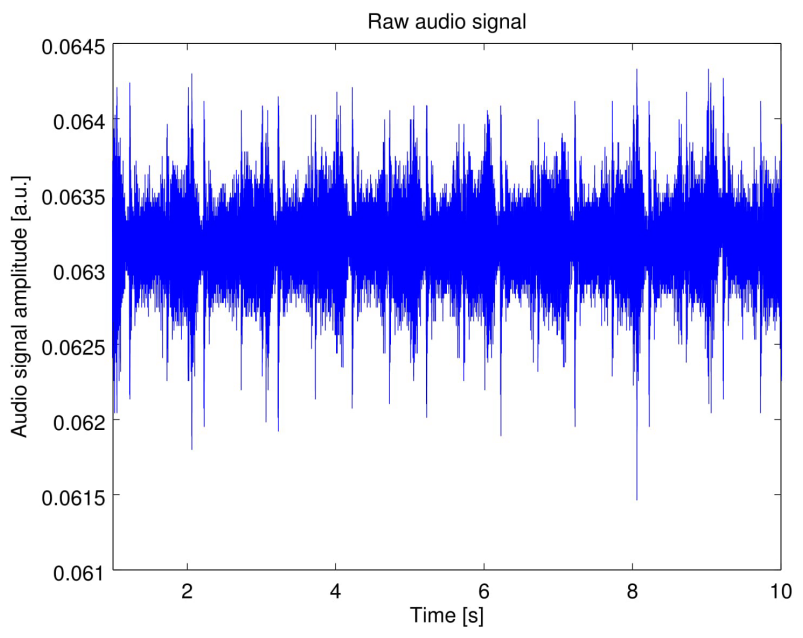

(a)

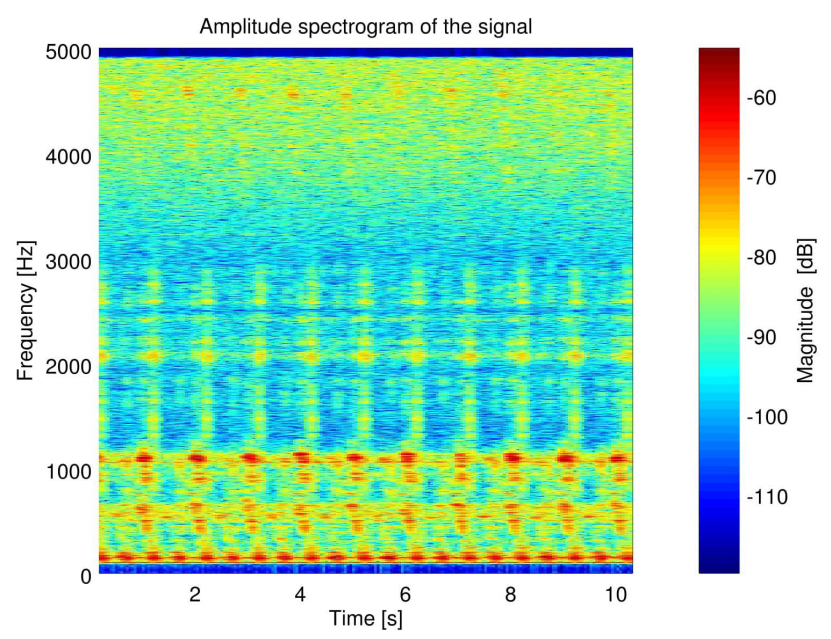

(b)

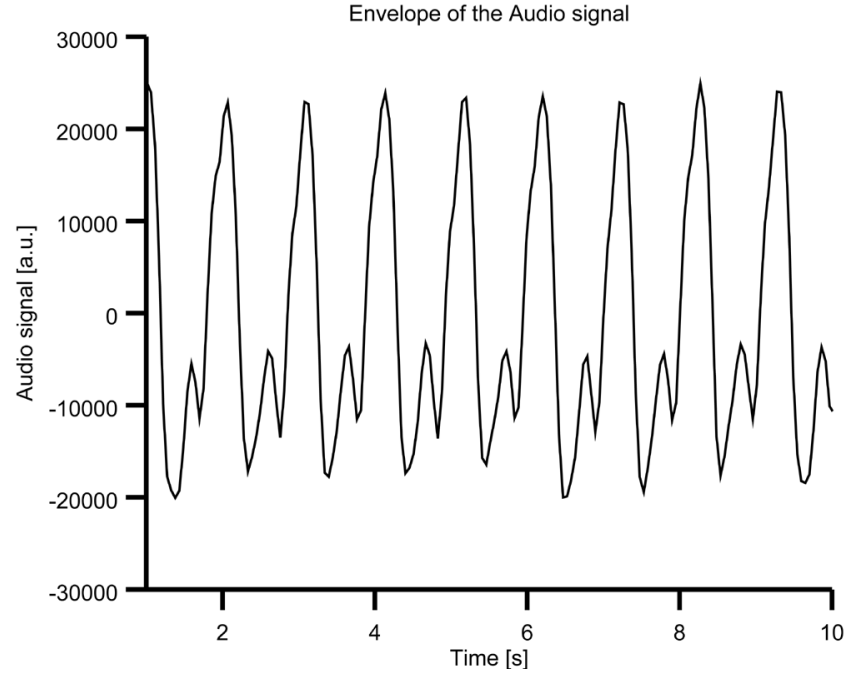

(c)

Figure 8. Measured vibrations and corresponding obtained signals at the coldhead (point A). (a) Signal obtained by piezo sensor; (b) STFT of (a); (c) Coldhead activity signal. 


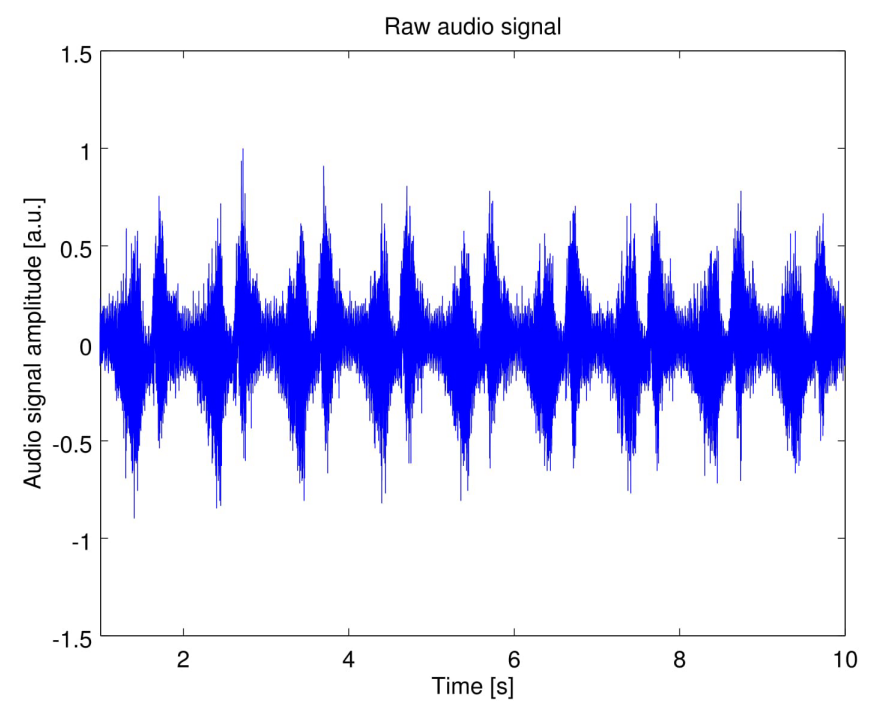

(a)

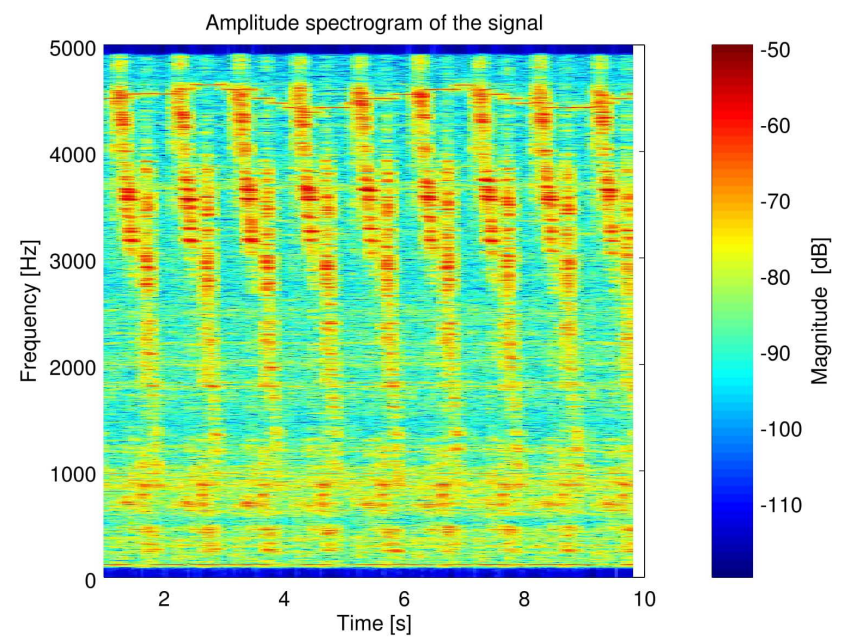

(b)

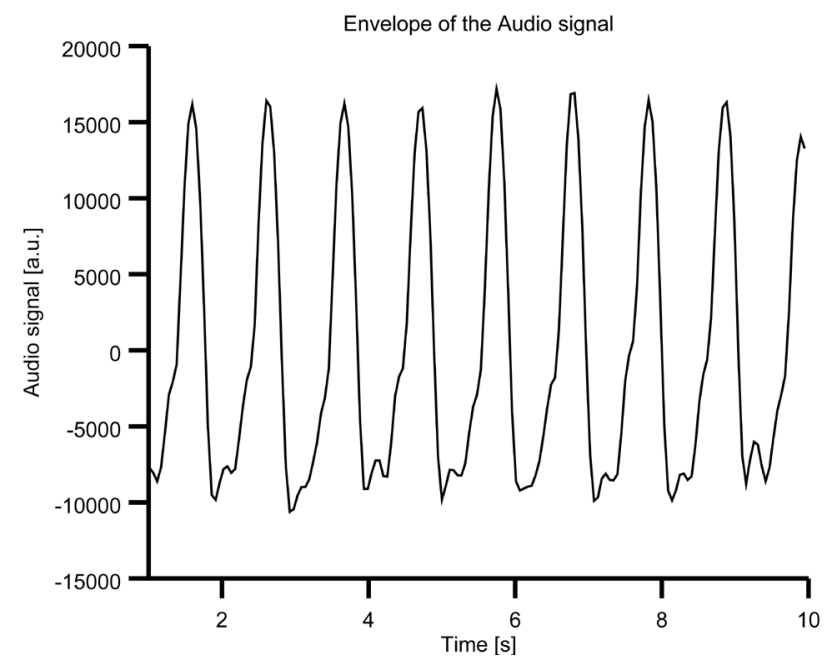

(c)

Figure 9. Measured vibrations and corresponding obtained signals at the gas pipe (point B). (a) Signal obtained by piezo sensor; (b) STFT of (a); (c) Coldhead activity signal. 


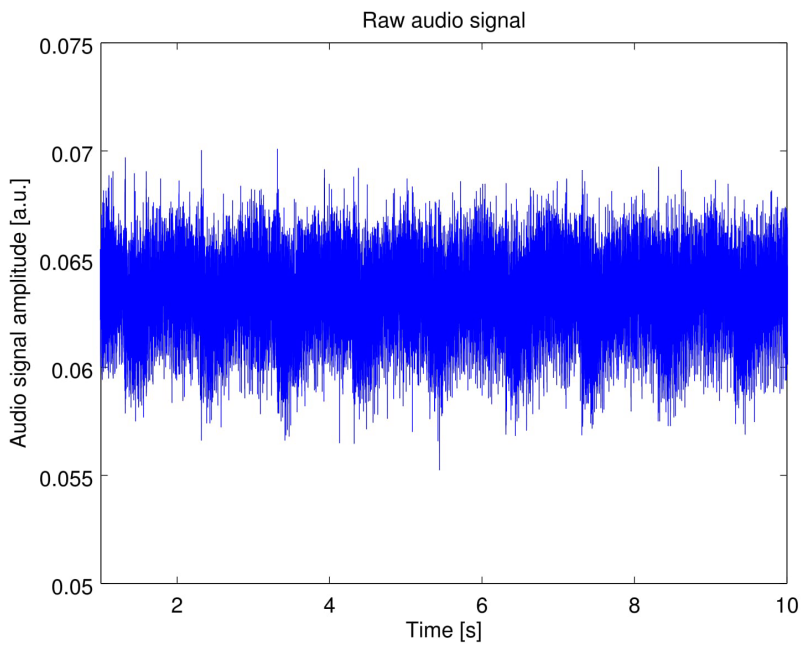

(a)

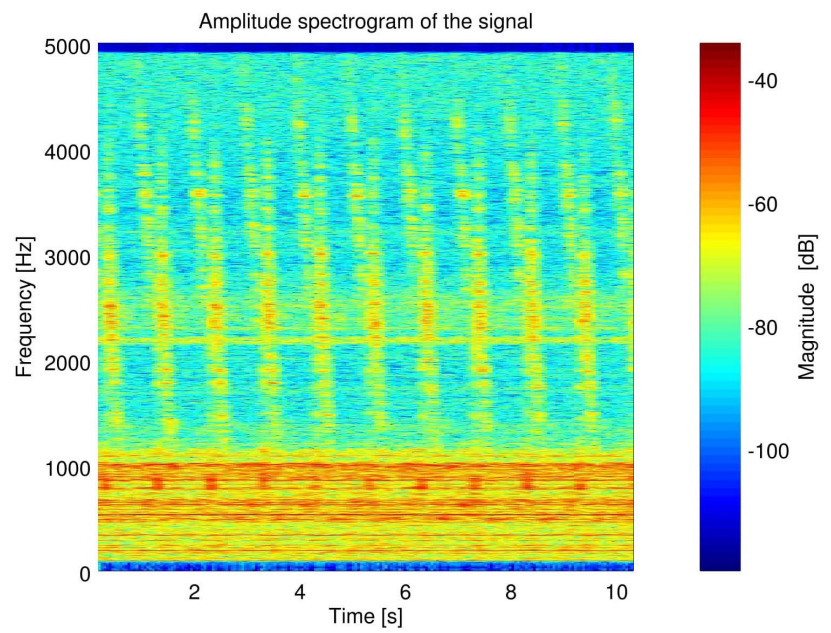

(b)

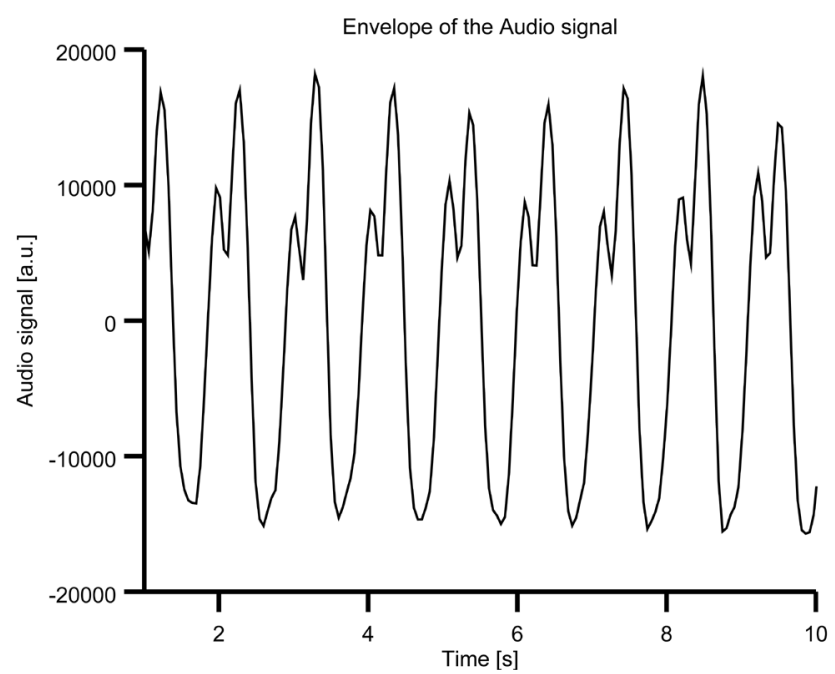

(c)

Figure 10. Measured vibrations and corresponding obtained signals at the gas pipe near the compressor (point D). (a) Signal obtained by piezo sensor; (b) STFT of (a); (c) Cold head activity signal. 


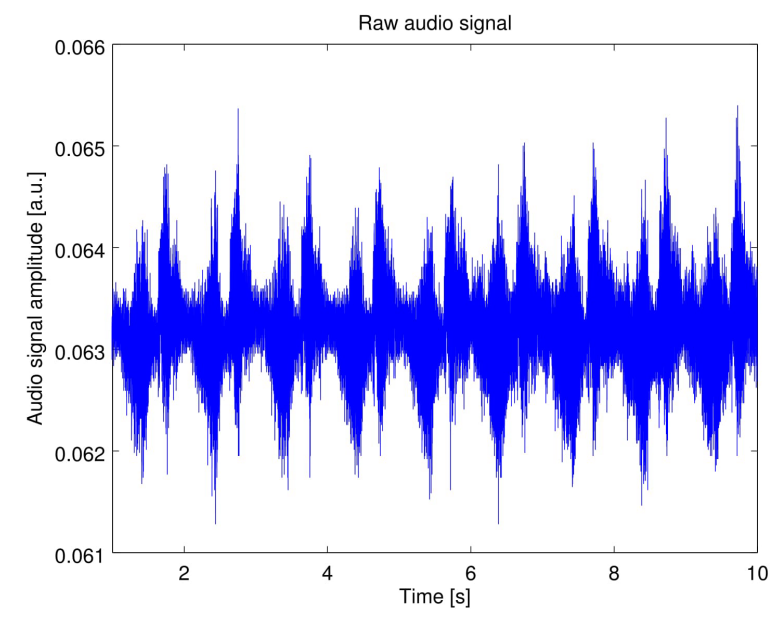

(a)

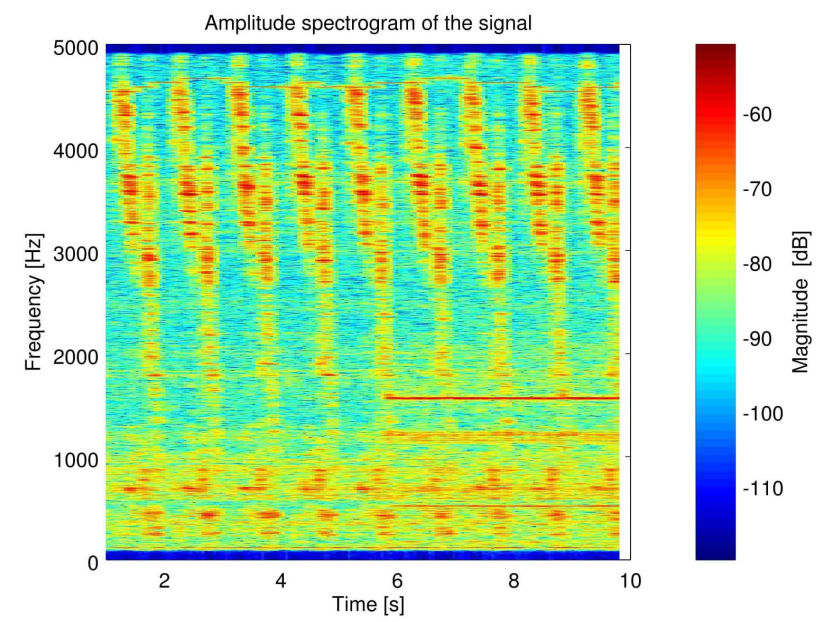

(b)

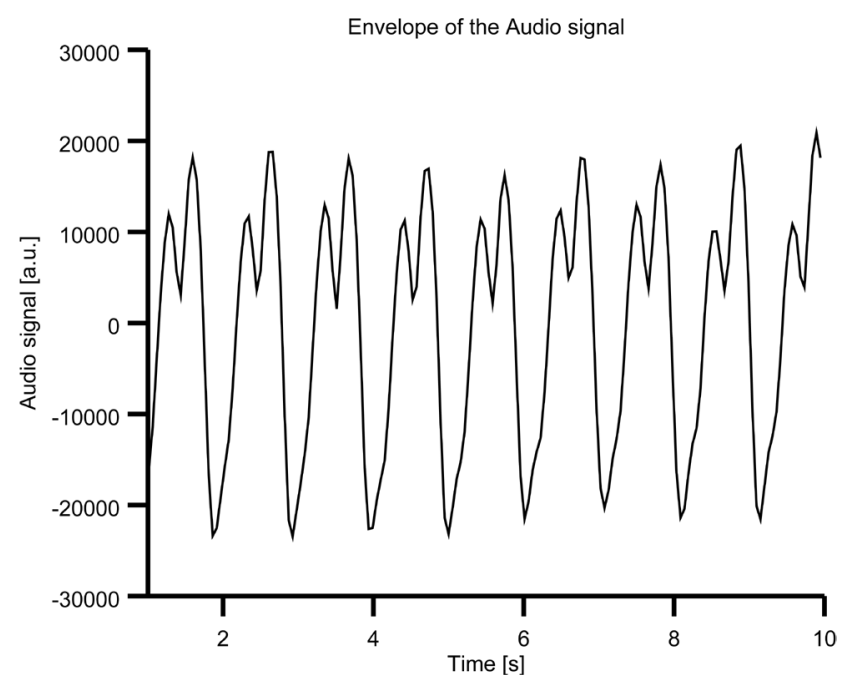

(c)

Figure 11. Measured vibrations and corresponding obtained signals at the gas pipe (point B) during a DTI sequence. Additional vibrations are visible in the frequency range between $1 \mathrm{kHz}$ and $2 \mathrm{kHz}$ in the time interval from $5 \mathrm{~s}$ to $10 \mathrm{~s}$. (a) Signal obtained by piezo sensor; (b) STFT of (a); (c) Coldhead activity signal. 
analyzed in this work were suitable for monitoring the coldhead's activity. The raw signal, the spectra and the extracted coldhead activity signal vary among the different locations. This observation can be explained by the fact that the helium supply lines as well as the exact sensor positions and placements act as filter structure which affects the acquired signals. Nevertheless, the vibrations caused by the coldhead were observable in all the analyzed locations. Although it was not shown here, a complete failure of the coldhead would result in a disappearance of the extracted coldhead activity signals or a power loss in the corresponding frequency bands. The $50 \mathrm{~Hz}$ vibrations of the helium compressor in the technical equipment room had no recognizable influence on the extracted signals. The additional noises or vibrations caused by the switched gradient magnetic fields did not disturb the extraction of the coldhead activity signals. This was shown with the examples given in Figures 8-11. Hence, for detecting if the coldhead is active, it is not required to perform the coldhead monitoring inside the MR scanner room. This is an important advantage since no electronic devices, cables or other components need to be place inside the sensitive environment of the MRI scanner.

The measurements which were made directly at the coldhead could provide additional information for a fault detection and diagnosis. The additional peaks observed in this signal are caused by different mechanical processes and components in the coldhead. Due to the limited amount of available data, i.e. due to the relatively short measurement duration, no clear statement can be made at this point about how this information can be used for analyzing the coldhead's wear or predicting a failure. However, it can be assumed that a detailed longterm analysis of the coldhead vibration signals enable a failure prediction. To establish such a measurement over a long period of time without interfering with signals of the MRI system, the currently used electrical RS422 connection needs to be replaced by an optical fiber link. In addition to the data transmission, the problem of energy supply needs to be solved. This could be either achieved by a battery driven system or an external power supply with a proper shield or filtering.

The long-term goal is to predict a failure of the MRI's coldhead. This requires a long-term monitoring of different coldheads over their whole life cycle to develop or train an algorithm allowing to detect such changes in the vibration signals. Sensor modules need to be placed at different MRI scanners or coldhead types at various locations (i.e. directly at the coldhead or in the adjacent technical room with the helium supply lines present) to investigate which locations and algorithms are best suited for performing this predictive analysis.

The low-cost piezoelectric element used in the current setup is limited by its resonant frequency. To extend the possible frequency range of the vibration measurement, a piezoelectric element with a higher resonant frequency should be used. This could reveal additional information contained in higher frequency components of the structure-borne noises. 


\section{Summary}

A method and corresponding experimental setup for monitoring an MRI's coldhead were proposed in this work. Using the developed systems and several measurements at a clinical MRI scanner system it was shown that the activity of a coldhead can be monitored using piezoelectric elements. Due to the transmission of the structure-borne noises, these measurements can be performed outside the MRI scanner cabin which has several practical advantages. The developed system will be used to perform a long-term monitoring of the mechanical vibrations in order to develop algorithms for assessing the current state of the coldhead's life-time and to predict possible failures. For such a failure prediction, it might be necessary to gather the additional information which is available directly at the coldhead. For that, additional hardware developments are required to perform the measurements directly at the cold head inside the shielded MRI scanner cabin. Once data is collected over longer period of time, i.e. several months or years, a retrospective analysis of this data can be used to develop algorithms for predicting failures or wear of the MRI's cold-head.

\section{References}

[1] Wilson, M.N. (1983) Superconducting Magnets. Clarendon Press, United Kingdom.

[2] Frey, H. and Haefer, R.A. (1981) Tieftemperaturtechnologie. VDI-Verlag, Düsseldorf.

[3] Gifford, W.E. (1965) The Gifford-McMahon Cycle. Advances in Cryogenic Engineering, $11,152-159$.

[4] Klein, U. (2000) Schwingungsdiagnostische Beurteilung von Maschinen und Anlagen. Verein für Betriebsfestigkeitsforschung. Verlag Stahleisen GmbH, Düsseldorf.

[5] Kuttner, T. (2015) Praxiswissen Schwingungsmesstechnik. Springer Vieweg, Leipzig.

[6] Bernstein, M.A., King, K.F and Zhou, X.J. (2004) Handbook of MRI Pulse Sequences. Elsevier, Amsterdam. 